\title{
NanoCrystalline Cellulose isolated from oil palm empty fruit bunch and its potential in cadmium metal removal
}

\author{
Yong Hui Lim ${ }^{1,2}$, Irene Mei Leng Chew ${ }^{3}$, Thomas Shean Yaw Choong ${ }^{1}$, Mei Ching $\operatorname{Tan}^{2}$ and Khang Wei Tan ${ }^{2}$ \\ ${ }^{1}$ Faculty of Engineering, University Putra Malaysia, 43400 UPM, Serdang, Selangor Darul Ehsan, Malaysia \\ ${ }^{2}$ Faculty of Engineering, Technology and Built Environment, UCSI University, Cheras, 56000 Kuala Lumpur, Malaysia. \\ ${ }^{3}$ School of Engineering, Monash University Sunway Campus, 46150 Bandar Sunway, Selangor Darul Ehsan, Malaysia.
}

\begin{abstract}
NanoCrystalline Cellulose (NCC) was isolated via ultrasonic cavitation assisted acid hydrolysis method. Characterization was done using Dynamic Light Scattering (DLS) together with Scanning Electron Microscope (SEM) imaging to double prove the existence of NCC. DLS measures length of $236.6 \mathrm{~nm}$ with width of $34.40 \mathrm{~nm}$, supported by SEM which showed NCC a rod-like shaped particle with large surface area and high porosity. It was then attempted for heavy metal cadmium ion $\left(\mathrm{Cd}^{2+}\right)$ removal from aqueous solution. The $\mathrm{pH}$ implication to the rate of $\mathrm{Cd}^{2+}$ adsorption was investigated by varying the solution to $\mathrm{pH} 4, \mathrm{pH} 7$ and $\mathrm{pH} 10$ over a duration of 120 minutes. The removal efficiency was analyzed using Atomic Absorption Spectroscopy (AAS) resulting in $\mathrm{pH} 7$ being the most favorable for $\mathrm{Cd}^{2+}$ removal.
\end{abstract}

\section{Introduction}

The growth of environmental issues has called forward to the search of new materials that are sustainable, biodegradable and environmental friendly [1]. Cellulose by far is the most abundant and inexhaustible natural resources which come with various benefits which has gain a lot of attention from researchers. Cellulose is available in various sources such as plant fibers and also lignocellulosic biomass. The biocompatibility towards human body and non-toxic nature were its winning points [2]. Cellulose can be decomposed into nanocrystalline cellulose (NCC) [3], which is a highly emerging bionanomaterial that offers many advantages i.e. high surface area, high aspect ratio, high specific strength together with stiffness, environmental benefits and low in cost [4].

Malaysia is an agricultural based tropical country with many industrial crops mainly the oil palm, paddy rice and sugarcane [5]. Malaysia the second largest oil palm producer with total oil palm plantation area up to 5.39 million hectares in 2014 (MPOB 2014). According to Malaysia Palm Oil Board (MPOB), about $22 \%$ of oil palm empty fruit bunch (EFB) was produced after fresh fruit bunch (FFB) processed in a palm oil mill. EFB contains high fibrous materials and packed with lots of lignocellulosic materials which can be source for cellulose. In 2013, it was reported that 8 million tonnes (dry weight) of EFB were produced annually making it a suitable raw material for the isolation of NCC.

Extensive reviews suggested that $\mathrm{NCC}$ has great potential in fields of medicine, optics, energy and composite materials. However, the applications of NCC in the following fields are still remain in the research stage. The negatively charged hydroxyl group on its surface giving opportunities for surface modification to its functionalization. Isolation of NCC via sulphuric acid hydrolysis method caused a negatively charge sulphate group to attach on its surface also acting as a stabilizer to suspense NCC in liquid such as water [6]. This suggests an opportunities for its application in drug delivery and waste water treatment in many industries.

Heavy metal pollution is one of the serious matters due to its toxicity not only towards human but also towards the ecosystem. Various heavy metal ions such as lead $(\mathrm{Pb})$, cadmium $(\mathrm{Cd})$, chromium $(\mathrm{Cr})$, copper $(\mathrm{Cu})$, nickel $(\mathrm{Ni})$, zinc $(\mathrm{Zn})$ and mercury $(\mathrm{Hg})$ are product of industrial wastewaters mainly from industry that processes metal plating, mining, and paint [7]. Substantial amounts of heavy metals was introduced into the atmosphere, aquatic and environment, leading to the increase of heavy metal exposure [8]. Heavy metals cannot be degraded by their own. They tend to accumulate themself in living organisms leading to various diseases and disorder. Lead and cadmium are considered the most toxic heavy metals ions which impose serious health issues [9]. High exposure from Lead can potentially lead to encephalopathy, cognitive impairment, behavioural disturbances, kidney damage, anaemia and toxicity to the reproductive system. Cadmium causes nephrotoxic effects. With its long term exposure, it may cause bone damage [8]. Based on World Health Organization (WHO), the permissible limit in 
waste water for lead is $0.015 \mathrm{mg} / \mathrm{L}$ and cadmium is 0.01 $\mathrm{mg} / \mathrm{L}$ [9].

Biosorption of heavy metals from aqueous solution is a promising process to remove contaminants from aqueous effluent [10]. Compared to conventional treatment, biosorption is low in cost, high efficient, recyclable, and possibility in the metal recovery [7]. A previous research had studied the feasibility of using NCC in waste water treatment, and the outcome is encouraging [9].

In the current study, NCC was isolated from oil palm biomass i.e. EFB using ultrasonic cavitation assisted acid hydrolysis. Sample characterization was done using Dynamic Light Scattering (DLS) measurement together with Scanning Electron Microscope (SEM). The extracted NCC was then employed to remove Cadmium ion from an aqueous solution. The mechanism of removal and the effect of $\mathrm{pH}$ against the performance of adsorption were investigated

\section{Methodology}

\subsection{Preparation of samples}

The pretreatment, bleaching and acid hydrolysis process to isolate NCC from Palm oil empty fruit bunch (EFB) via a conventional method was published in previous work [11]. Briefly, EFB was dried and grinded using a household grinder before pass screening through a sieve of $300 \mu \mathrm{m}$. The sieved EFB was first washed using distilled water at a temperature of $50^{\circ} \mathrm{C}$ for $2 \mathrm{hr}$ to remove dust and impurities together with waxy substances on the surface followed with oven-drying at $70^{\circ} \mathrm{C}$ for $24 \mathrm{hr}$. The oven-dried EFB was then treated with $4 \% \mathrm{NaOH}$ solution at temperature of $80^{\circ} \mathrm{C}$ for $6 \mathrm{hr}$ and washed thoroughly using distilled water until $\mathrm{pH} 7$ was reached. This process is known as alkaline treatment, repeated twice using the same setting and then proceeded with oven-drying for $24 \mathrm{hr}$ at $70^{\circ} \mathrm{C}$ temperature. $30 \%$ hydrogen peroxide $\left(\mathrm{H}_{2} \mathrm{O}_{2}\right)$ solution with few drops of glacial acetic acid was further applied on the alkalinetreated EFB for $7 \mathrm{hr}$ as the delignification or bleaching process. After washing it thoroughly until $\mathrm{pH} 7$ was reached, it was proceed to oven-drying for $24 \mathrm{hr}$ at $70^{\circ} \mathrm{C}$.

\subsection{Isolation of nanocrystalline cellulose}

Bleached EFB was dispersed in $60 \%$ sulphuric acid $\left(\mathrm{H}_{2} \mathrm{SO}_{4}\right)$ solution at a constant temperature of $45^{\circ} \mathrm{C}$ for 2 $\mathrm{h}$. The acid used to hydrolyze the amorphous region of the cellulose, isolating only the crystalline region cellulose, which is less susceptible to acid attack. Once the reaction completed, cold distilled water was introduced to quench the reaction, bringing the acid hydrolysis to a stop. The post-reaction liquid was then repeatedly centrifuged for $15 \mathrm{~min}$ at 7,000 rpm, to obtain raw NCC suspension. This process was repeated till an inseparable suspension is formed. The suspension was filtered and washed with distilled water until $\mathrm{pH} 7$ was reached. The filtrate was then dispersed in distilled water.

\subsection{Ultrasonic cavitation}

NCC suspended in distilled water was subjected to ultrasonic cavitation treatment using KUDOS ultrasonic bath model SK7210HP at power of $100 \%$ with frequency of $53 \mathrm{kHz}$ for $15 \mathrm{~min}$. Ultrasonic cavities created by ultrasonic bath further break down the crystalline cellulose into nano-size known as NCC. The NCC produced was left dispersed in water for further characterization and application as described next.

\subsection{Dynamic light scattering measurement}

Particles size of NCC were measured using Dynamic Light Scattering (DLS) method with Malvern Zetasizer Nano ZS as described in [1]. The measurement was repeated twice to obtain the average value for $\mathrm{z}$-average.

\subsection{Cadmium metal ions removal}

Heavy metal ions was removed from aqueous solution by adsorption via NCC as described in [9] with slight modification. In this study, a $500 \mathrm{~mL}$ standard solution of $1 \mathrm{mg} / \mathrm{L}$ cadmium was mixed with $1 \mathrm{~mL}$ of $\mathrm{NCC}$ suspension and stirred at $120 \mathrm{rpm}$ for $2 \mathrm{hr}$. The $\mathrm{pH}$ of the solution is adjusted using $\mathrm{NaOH}$ and HCI solution to obtain $\mathrm{pH} 4, \mathrm{pH} 7$, and $\mathrm{pH} 10$. Samples were collected every $20 \mathrm{~min}$ and analyzed using Atomic Absorption Spectroscopy (AAS, Pelkin Elmer AAnalysis 400) to determine the percentage of cadmium ions removal.

\section{Results and Discussion}

\subsection{Isolation of nanocrystalline cellulose}

The EFB was pre-treated with mechanically disintegration, washing and drying prior to alkaline treatment and delignification process, before the last stage cellulose isolation. Theoretically, lignin, hemicellulose, wax and oils on the surface of cellulose can be effectively removed via alkaline treatment which helps to swell the cellulose, defibrillates the cellulose, and exposing the crystallites [12]. Delignification or bleaching process further the alkaline treatment and gives a bleaching effect on the treated EFB. It can be observed from the colour change i.e. from brown to white, indicating a decrease in percentage of hemicellulose and lignin from EFB [13]. After that, isolation of NCC driven by sulphuric acid hydrolyse the amorphous region due to its high reactivity via cleavage of the cellulose glycosidic bonds, and the introduction of hydrophilic sulphate ($\left.\mathrm{OSO}_{3} \mathrm{H}\right)$ groups to the crystalline cellulose [14]. This suggest the formation of negatively charged NCC that induce electrostatic repulsion forces among NCC particles making the aqueous suspension stable [15]. The NCC usually range in length from $100 \mathrm{~nm}$ to $3000 \mathrm{~nm}$ for many cellulosic materials and individualized by ultrasonic cavitation. The dimensions of nanocrystalline 
cellulose are affected by the source of cellulose and hydrolysis conditions [16].

\subsection{Dynamics Light Scattering measurement}

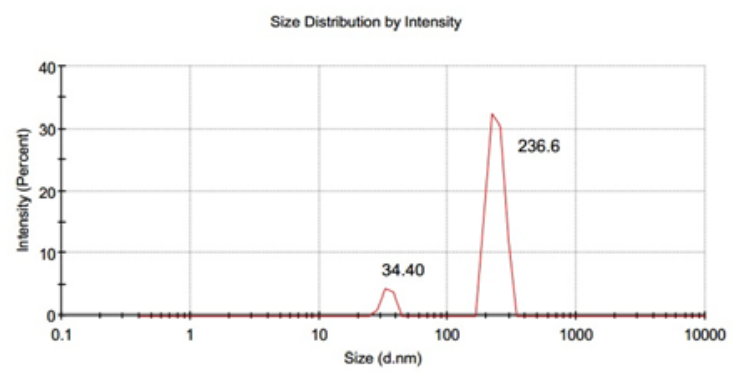

Figure 1. Particle size analysis using Dynamic Light Scattering Measurement using Malvern Zetasizer, Nano ZS Series.

DLS is a noninvasive method of measuring particle dispersed in a medium. Particles in dispersion will undergo Brownian motion and DLS measures the translational diffusion coefficient of the particle in the dispersion [2]. Figure 1 shows the results of DLS which indicates two size distribution intensity represented by the two peaks. An average size, z-average diameter was obtained as $561.0 \mathrm{~nm}$. Peak 1 recorded particle size of $236.6 \mathrm{~nm}$ and peak 2 recorded particle size of $34.40 \mathrm{~nm}$ which can be interpret to the corresponding length and the width of the NCC. NCC has a rod-like shaped as described by various reviews, with length ranging from $100 \mathrm{~nm}$ to $3000 \mathrm{~nm}$ and width ranging from $3 \mathrm{~nm}$ to 30 $\mathrm{nm}$ using imaging technique of measurement such as atomic field microscope (AFM) and transmission electron microscope (TEM). Based on Stoke-Einstein theory, larger particle moves slower in a medium as compared to smaller particle which diffuses at a greater rate [17]. In this case, if NCC diffuses across the aqueous medium in a horizontal orientation against the light source, DLS would probably interpret it as a smaller particle due to its higher diffusion speed. Likewise, if NCC diffuses across the aqueous medium in a vertical orientation against the light source, DLS would most probably interpret it a bigger particle due to its slower diffusion rate as a result of higher resistance received toward the moving direction. Concisely, the orientation of particles scattered the light in different angle outlined two peaks of information that reflect the NCC diffusion orientation. The DLS measurement proved the existence of the NCC and a Scanning Electron Microscope (SEM) imaging was done to further support the result.

\subsection{Scanning Electron Microscope}

The morphology of the extracted NCC was determined using SEM imaging. NCC was dried before proceeding to SEM imaging. Figure 2 shows rod-like shaped NCC on the bottom right while some rod-like NCC clustered or bundled on at the top left corner of the image, in line with a previous study [18]. The length and width was confirmed. As from the SEM image, NCC is found to have large surface area and the bundles which looks like the stacking of the NCC, produced network-structure. The network structure also found to be the result of NCC in overcoming the repulsion of the surface negative charges which led to formation of self-assembled porous networks [18]. This phenomena brings to the high porosity properties of NCC that would benefit in the heavy metal removal. High surface area and high porosity properties can provides more active sites for the adsorption and desorption ions.

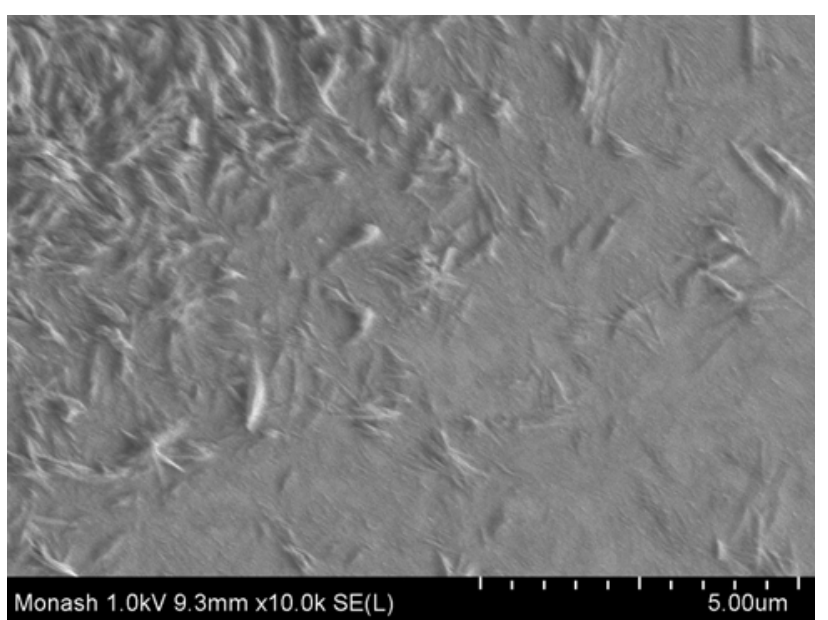

Figure 2. SEM imaging of NCC at 10,000 X magnification.

\subsection{Cadmium removal}

A similar research was reported [9] by functionalizing NCC into sodic NCC (NaSCNCs). The NaSCNCs acts as nanoadsorbent in removing $\mathrm{Pb}^{2+}$ and $\mathrm{Cd}^{2+}$, and it is able to regenerate with $\mathrm{NaCl}$ solution. These suggested that the chemically modified cellulose could potentially achieve efficient adsorption capacity of heavy metal ions as stated by [19]. The cellulose can adsorb metal ions via ion exchange which contributed from the active sites present on them. NCC as reported earlier was found to have great surface area exposed and high porosity making it more susceptible to absorb more metal ions.

Solution pH plays a major role in heavy metal removal as it affects the activity of the functional groups of the sorbent and the metal ions [20]. As in this case, which is the hydroxl $(\mathrm{OH})$ group on the surface of NCC and the $\mathrm{Cd}^{2+}$. Figure 3 shows the comparison of the cadmium removal rate at $\mathrm{pH}$ value of 4,7 and 10 . It shows highest rate of removal at $\mathrm{pH} 7$ which is almost the same as reported in [20] and [21]. As expected in lower $\mathrm{pH} 4$, there are lesser ligands available for metal ions to binding due to the high concentration of hydrogen ions $\left(\mathrm{H}_{3} \mathrm{O}^{+}\right.$, hydronium $)$that suggest competition between hydronium and metal ions for the free binding sites. With increase of $\mathrm{pH}$, lesser hydronium present, deprotonate the binding sites on the surface making more ligand groups available for metal ion bonding. The electrostatic attraction between the hydroxide group and the free electron-pairs from cadmium ions enhanced [21]. The increase of $\mathrm{pH}$ also indicated that the surface became 
negatively charged, making cations adsorption (which in this case the $\mathrm{Cd}^{2+}$ ) more favorable [22].

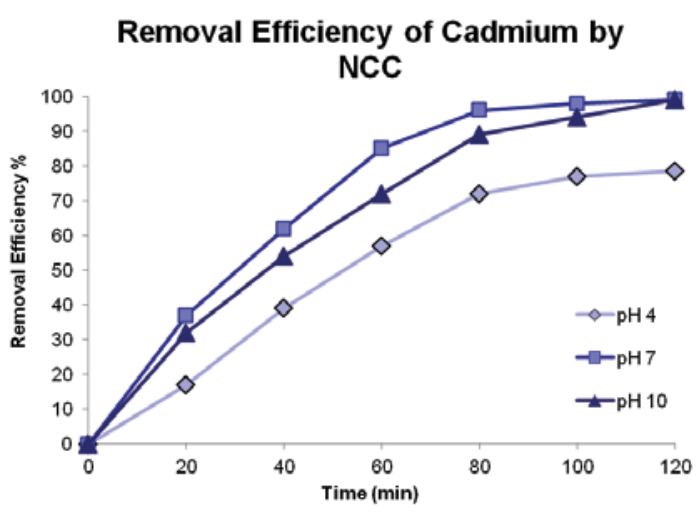

Figure 3. Cadmium metal ions removal efficiently by NCC.

\section{Conclusion}

NanoCrystalline Cellulose (NCC) was successfully isolated from palm oil empty fruit bunch (EFB) using ultrasonic cavitation assisted acid hydrolysis method. The Dynamic Light Scattering (DLS) measurements gave two peaks readings at $34.40 \mathrm{~nm}$ and $236.6 \mathrm{~nm}$ which interpreted as the diameter and length measurement respectively. The unique properties of NCC isolated from acid hydrolysis using sulphuric acid, which is the sulphate bonding on NCC surface promote the adsorption of cadmium in the application of heavy metal ions removal. As the result $\mathrm{pH}$ effect, it was found that $\mathrm{NCC}$ showed optimal cadmium ions removal rate at $\mathrm{pH} 7$, which is at neutral state.

\section{Acknowledgement}

The authors are thankful to the Ministry of Higher Education, Malaysia (MoHE) for providing financial support (ERGS) on this research. Credit also goes to University Putra Malaysia, UCSI University Malaysia and Monash University Malaysia Campus for various infrastructures provided for advanced analysis work in this research field.

\section{References}

1. Khouri, S., Shams, M., \& Tam, K. C. (2014). Determination and prediction of physical properties of cellulose nanocrystals from dynamic light scattering measurements. Journal of Nanoparticle Research, 16 (7). http://doi.org/10.1007/s11051-0142499-7.

2. Boluk, Y., \& Danumah, C. (2014). Analysis of cellulose nanocrystal rod lengths by dynamic light scattering and electron microscopy. Journal of Nanoparticle Research, 16 http://doi.org/10.1007/s11051-013-2174-4.

3. Klemm, D., Kramer, F., Moritz, S., Lindström, T., Ankerfors, M., Gray, D., \& Dorris, A. (2011).
Nanocelluloses: A new family of nature-based materials. Angewandte Chemie - International Edition, $\quad \mathbf{5 0} \quad \mathbf{( 2 4 ) ,}$ 5438-5466. http://doi.org/10.1002/anie.201001273.

4. Abdul Khalil, H. P. S., Davoudpour, Y., Islam, M. N., Mustapha, A., Sudesh, K., Dungani, R., \& Jawaid, M. (2014). Production and modification of nanofibrillated cellulose using various mechanical processes: a review. Carbohydrate Polymers, 99, 649-65. http://doi.org/10.1016/j.carbpol.2013.08.069.

5. Hosseini, S. E., \& Wahid, M. A. (2013). Feasibility study of biogas production and utilization as a source of renewable energy in Malaysia. Renewable and Sustainable Energy Reviews, 19, 454-462. http://doi.org/10.1016/j.rser.2012.11.008.

6. Moon, R. J., Martini, A., Nairn, J., Simonsen, J., \& Youngblood, J. (2011). Cellulose nanomaterials review: structure, properties and nanocomposites. Chemical Society reviews 40. http://doi.org/10.1039/c0cs00108b.

7. Demirbas, A. (2008). Heavy metal adsorption onto agro-based waste materials: A review. Journal of Hazardous Materials, 157 (2-3), 220-229. http://doi.org/10.1016/j.jhazmat.2008.01.024.

8. O'Connell, D. W., Birkinshaw, C., \& O'Dwyer, T. F. (2008). Heavy metal adsorbents prepared from the modification of cellulose: A review. Bioresource Technology, $99 \quad$ (15), $6709-6724$. http://doi.org/10.1016/j.biortech.2008.01.036.

9. Yu, X., Tong, S., Ge, M., Wu, L., Zuo, J., Cao, C., \& Song, W. (2013). Adsorption of heavy metal ions from aqueous solution by carboxylated cellulose nanocrystals. Journal of Environmental Sciences, 25 (5), 933-943. http://doi.org/10.1016/S10010742(12)60145-4.

10. L.Y.Lee, X.J.Lee, P.C.Chia, K.W.Tan and S.Gan (2014) Utilization of Cymbopogon citratus (lemon grass) as biosorbent for the sequestration of nickel ions from aqueous solution: Equilibrium, kinetic, thermodynamics and mechanism studies, Journal of the Taiwan Institute of Chemical Engineers 45, 1764-1772. doi:10.1016/j.jtice.2014.02.002.

11. Shanmugarajah B., Kiew P. L., Chew M. L., Chong S. Y., Tan K.W. (2015) Isolation of nanocrystalline cellulose (NCC) from palm oil empty fruit bunch (EFB): Preliminary result on FTIR and DLS analysis. Chemical Engineering Transactions, 45, 1705-1710. http://doi:10.3303/CET1545285.

12. Abraham, E., Deepa, B., Pothan, L. a., Jacob, M., Thomas, S., Cvelbar, U., \& Anandjiwala, R. (2011). Extraction of nanocellulose fibrils from lignocellulosic fibres: A novel approach. Carbohydrate Polymers, 86(4), 1468-1475. http://doi.org/10.1016/j.carbpol.2011.06.034

13. Lu, P., \& Hsieh, Y.-L. (2012). Preparation and characterization of cellulose nanocrystals from rice straw. Carbohydrate Polymers, 87(1), 564-573. http://doi.org/10.1016/j.carbpol.2011.08.022

14. Hu, T. Q., Hashaikeh, R., \& Berry, R. M. (2014). Isolation of a novel, crystalline cellulose material from the spent liquor of cellulose nanocrystals 
(CNCs). Cellulose, 3217-3229. http://doi.org/10.1007/s10570-014-0350-Z

15. Mueller, S., Weder, C., \& Foster, E. J. (2014). Isolation of cellulose nanocrystals from pseudostems of banana plants. RSC Advances, 4(2), 907. http://doi.org/10.1039/c3ra46390g

16. Sheltami, R. M., Abdullah, I., Ahmad, I., Dufresne, A., \& Kargarzadeh, H. (2012). Extraction of cellulose nanocrystals from mengkuang leaves (Pandanus tectorius). Carbohydrate Polymers, 88(2), 772-779.

http://doi.org/10.1016/j.carbpol.2012.01.062

17. Sharma, M., \& Yashonath, S. (2007). Size dependence of solute diffusivity and Stokes-Einstein relationship $\square$ : effect of van der Waals interaction. Diffusion Fundamentals, 7, 1-15.

18. Tang, Y., Yang, S., Zhang, N., \& Zhang, J. (2013). Preparation and characterization of nanocrystalline cellulose via low-intensity ultrasonic-assisted sulfuric acid hydrolysis. Cellulose, 21(1), 335-346. http://doi.org/10.1007/s10570-013-0158-2

19. Kamel, S., Hassan, E. M., \& El-Sakhawy, M. (2006). Preparation and application of acrylonitrile-grafted cyanoethyl cellulose for the removal of copper (II) ions. Journal of Applied Polymer Science, 100 (1), 329-334. http://doi.org/10.1002/app.23317.

20. Wei, W., Kim, S., Song, M.-H., Bediako, J. K., \& Yun, Y.-S. (2015). Carboxymethyl cellulose fiber as a fast binding and biodegradable adsorbent of heavy metals. Journal of the Taiwan Institute of Chemical Engineers, $\quad 57, \quad 1-7$. http://doi.org/10.1016/j.jtice.2015.05.019.

21. Gurgel, L. V. A., \& Gil, L. F. (2009). Adsorption of $\mathrm{Cu}(\mathrm{II}), \mathrm{Cd}(\mathrm{II})$ and $\mathrm{Pb}(\mathrm{II})$ from aqueous single metal solutions by succinylated twice-mercerized sugarcane bagasse functionalized with triethylenetetramine. Water Research, 43 (18), 44794488. http://doi.org/10.1016/j.watres.2009.07.017.

22. Guo, H., Zhang, S., Kou, Z., Zhai, S., Ma, W., \& Yang, Y. (2015). Removal of cadmium(II) from aqueous solutions by chemically modified maize straw. Carbohydr Polym, 115, 177-185. http://doi.org/10.1016/j.carbpol.2014.08.041. 